\title{
Editorial
}

\section{The dilemma of the epidural test dose}

The epidural test dose was first recommended by Dogliotti in 1933.' To avoid the complication of a high spinal block he suggested injecting a small volume of local anaesthetic through the needle in the epidural space to insure that a subarachnoid placement had not occurred prior to injecting the full epidural dose. Anaesthetists were already well aware of the dangers of the high spinal block, becausc the technique had enjoyed a brief period of popularity, until severe respiratory and cardiovascular effects were reported in some surgical patients. ${ }^{2}$

With the introduction of epidural anaesthesia into obstetrics, the test dose was also recommended for the "single-shot" technique. Total spinal blocks still occurred occasionally but rapid resuscitation by the anaesthetist usually resulted in a good outcome. ${ }^{3}$ This was likely due to the fact that the anaesthetist was physically present in the room when the complication occurred.

With the introduction of the epidural catheter, the situation became morc complicated. Should the test dose be injected through the needle or through the catheter? Some recommended injection through the needle, because this then served the dual purpose of testing for subarachnoid placement of the needle and enlarging the epidural space to make passage of the catheter easier. Other anaesthetists pointed out that the test dose should be injected through the catheter since the catheter would be used subsequently to inject the remainder of the dose of local anaesthetic. There were no studies reported comparing these two methods, probably because the incidence of total spinal block was so low that only a very large series would demonstrate which technique was superior.

As the use of epidural anaesthetics in obstetrical patients became more popular, the continuous analgesia technique for the labouring patient was described. In Bonica's original publication ${ }^{4}$ and in studies from other centres, ${ }^{3,5.6}$ the incidence of total spinals was extremely low. Individual case reports appeared in the literature but adverse outcomes were rare. ${ }^{7}$ Some authors described a

Department of Anesthesia, St. Boniface Gencral Hospital, Unjversity of Manitoba, 409 Tache Avenue, Winnipcg, Manitaba, R2H 2 A6. technique for a test dose; others did not think it was warranled.

The problem of test doses for "top-ups" was seldom discussed. The reliability of test doses was questioned, because a partial block would be in place from the previous injection. Major complications, although still rare, became associated with more adverse outcomes with top-ups being done by nurses and midwives, without the anaesthetist being present in the room. ${ }^{9}$ In analyzing these events several questions arose which stimulated a great deal of discussion about the safest way to provide continuous analgesia for the labouring patient. Test doses were recommended by some but there was no agreement as to which local anaesthetic should be used, or in what volume and concentration. This issue still remains to be resolved.

In the late seventies and early eighties another problem surfaced related to the local anaesthetic agent bupivacaine. This was the consequences of the intravascular injection of the local anaesthetic. ${ }^{10}$ This problem had been previously described with lidocaine but because it was rare and seldom accompanied by an adverse outcome it had not generated much discussion. The cardiotoxicity of bupivacaine, however, resulted in several maternal deaths and produced many reports concerning the mechanism of cardiovascular collapse seen with this complication. ${ }^{11,12}$ In attempting to prevent this serious problem, the test dose was advocated to serve as a waming of an intravascular injection. It was pointed out that small doses of local anaesthetic injected intravenously might not produce signs and symptoms in young healthy patients. Therefore the test dose for detecting a subarachnoid injection might not rule out an intravascular injection. Moore and Batra ${ }^{13}$ recommended adding epinephrine to the test dose and monitoring the ECG to detect an increase in the heart rate which would occur if the epinephrine was injected into an epidural vein. They recommended using $15 \mu \mathrm{g}$ of epinephrine. Subsequent publications have disagreed with this approach because of (1) possible deleterious effects of epinephrine on uterine blood flow ${ }^{14}$ and (2) the difficulty with interpretation of heart rate changes in labouning patients who may manifest 20-30 per cent changes in heart rate with contractions. ${ }^{15}$ 
The review article in this issue by Dain, Rolbin and Hew discusses in detail the problems of selecting and interpreting an epidural test dose. I agree with the authors' conclusion that lidocaine is the best choice of local anaesthetic currently available. Lidocaine will demonstrate a motor and sensory block in a shorter time than bupivacaine if injected into the subarachnoid space. I also think that a hyperbaric solution of lidocaine would, if available, be safer in terms of a limited spread in the subarachnoid space. The use of the test dose to demonstrate an intravascular injection is much less reliable and the use of epinephrine as a marker may be confusing in a labouring patient.

Like Dain et al. I do use test doses. I consider the test dose to be an indicator primarily of a subarachnoid injection. Currently, after a negative aspiration test, 1 inject $2.0 \mathrm{ml}$ of two per cent lidocaine through the catheter, wait five minutes and then check for both motor and sensory block. Blood pressure and heart rate are monitored at two-minute intervals. I also question the patient about any sensation of circumoral numbness or tinnitis as an indication of an intravascular reaction knowing that if these symptoms do not occur the intravascular injection has not necessarily been ruled out.

If no adverse signs or symptoms occur then I inject either carbonated lidocaine for Caesarean section or bupivacaine 0.25 per cent for labouring patients, in volumes of 5-6 ml at five-minute intervals. This partitioning of the block dose is a further safeguard against subarachnoid or intravascular injection of large amounts of local anaesthetic. A satisfactory block should occur within 20 minutes or misplacement of the catheter is highly likely.

To specifically guard against intravascular injection I do not inject catheters that contain blood, either appearing spontaneously or after aspiration of the catheter. If blood has been obtained then I remove the catheter and repeat the technique at another interspace. If no blood is obtained at the second insertion then I do add epinephrine $15 \mu \mathrm{g}$ to the test dose and monitor the pulse rate either by ECG or a pulse oximeter. If there is no pulse rate increase in excess of 20 beats $\cdot \mathrm{min}^{-1}$ in five minutes 1 then proceed as above.

For labouring patients having "top-ups," test doses are less likely to reveal a problem. To guard against intravascular or subarachnoid injection, I partition the dose so that the volume injected is never more than $5 \mathrm{ml}$ at a time with a 3-5 minute waiting period between each injection. With every injection of an epidural there must be constant observation of the patient. Vital signs must be monitored at one to two minute intervals for at least ten minutes following each injection and signs of motor and sensory block must be actively sought.

With epidural anaesthesia or analgesia in the obstetrical patient there is no substitute for close observation of the patient by someone trained to detect adverse effects. The test dose may help the trained observer to detect subarachnoid or intravascular injections but should never be considered to replace careful observation during the conduct of epidural anaesthesia. Because subarachnoid and intravascular injection of local anaesthetic are rare during the conduct of epidural anaesthesia, many anaesthetists may be lulled into the thought that they do not occur at all and that elaborate precautions are unnecessary. This may result in severe morbidity or mortality for the one case where either complication occurs. Epidural anesthesia will only be safe for all patients if everyone has a healthy respect for these rare but severe complications.

\section{Le dilemne de la dose epidurale d'essai}

La dose épidurale d'essai fut initialement recommandéc par Dogliotti en 1933.' Afin d'éviter les complications d'un haut blocage spinal il suggéra d'injecter un petit volume d'anesthésique local à travers l'aiguille dans l'espace épidural afin de s'assurer que la localisation de l'aiguille sous-arachnoïdienne ne soit survenue avant l'injection de la dose totale requise pour l'épidurale. Les anesthésistes étaient déjà conscients du danger d'un haut bloc spinal et la technique fut si populaire pour une courte période jusqu'à ce que ses effets sévères tant respiratoires que cardiovasculaires soient rapportés chez certains patients devant subir la chirurgie. ${ }^{2}$

Avec l'introduction de l'anesthésie épidurale en obstétrique, la dose d'essai a été aussi recommandée pour la technique épidurale à dose unique. Une "rachi totale" survenait occasionnellement et une ressuscitation rapide par l'anesthésiste aboutissait souvent à une issue favorable. ${ }^{3}$ Ceci était probablement dû au fait que l'anesthésiste était physiquement présent lors de la survenue de la complication.

Avec l'introduction du cathéter épidural la situation se compliqua. Doit-on injecter la dose d'essai à travers l'aiguille ou à travers le cathéter? Certains recommandèrent l'injection à travers l'aiguille car ceci servirait à la fois à détecter son emplacement sous-arachnoïdien et à élargir l'espace épidural pour le passage facile du catheter. D'autres anesthesistes ont recommandé l'injection de la dose d'essai à travers le cathéter car ce dernier serait utilisé subséquemment pour l'injection de la balance de la 
dose de l'agent anesthésique local. Il n'y eût aucunétude de rapportée comparant ces deux méthodes, probablement parce que l'incidence de rachi totale était si basse qu'il serait nécessaire d'avoir une très large série afin de démontrer la superiorité de l'une ou l'autre des deux méthodes.

Avec la popularité croissante de l'épidurale en obstétrique, la technique d'anal gésie continue lors du travail fut décrite. Dans la publication ${ }^{4}$ originale de Bonica ainsi que dans d'autres études provenant de différents centres, ${ }^{3,5,6}$ l'incidence de rachi totale était extrêmement basse. Des cas isolés furent rapportés dans la littérature mais les issues défavorables étaient rares. ${ }^{7}$ Certains auteurs ont décrit une technique pour la dose d'essai; d'autres ne la croyaient pas justifiée.

Le problème des doses d'essai pour des "rajouts" était rarement discuté. La fiabilité des doses d'essai était discutable à cause de la présence d'un bloc pariel par l'injection précédente. Les complications majeures même si encore rares étaient de plus en plus associées à des issues défavorables car les doses de "rajout" étaient administrées par des infirmières et sage-femmes, en l'absence d'un anesthésiste physiquement présent dans la salle. ${ }^{9}$ En analysant ces évènements plusieurs questions furent soulevées stimulant ainsi un grand nombre de discussions concernant la façon la plus sêcure d'offrir une analgésie continue lors du travail. Les doses d'essai furent recommandées par certains sans que pour autant il en résultẹ un concensus sur l'agent anesthésique local, ou son volume ou sa concentration. Ce problème demeure encore à résoudre.

A la fin des années 1970 et vers le début des années 1980 un autre problème surgit en rapport avec la bupivacaïne. Ceci fut la conséquence de l'injection intravasculaire de l'anesthésique local. ${ }^{10} \mathrm{Ce}$ problème fut préalablement décrit avec la lidocaine et le fait qu'il fut peu fréquent et rarement accompagné d'issue défavorable il n'engendra pas autant de discussion. La cardiotoxicité de la bupivacaïne occasionna cependant plusieurs décès matcrncls et engendra plusicurs rapports concernant le mécanisme du collapsus cardiovasculaire observé avec cette complication. ${ }^{11.12}$ Afin d'essayer de prevenir ce problème sérieux, les doses d'essai furent suggérées comme moyen d'avertissement d'une injection intravasculaire. Il a été noté que de petites doses d'anesthésique local injectées dans la veine peuvent ne pas produire des signes et symptômes chez les patients jeunes et sains. Ainsi la dose d'essai pour détecter une injection sousarachnoidienne n'élimine pas une injection intrayascu. laire. Moore et Batra ${ }^{13}$ ont recommandé l'addition d'épinéphrine à la dose d'essai et de monitoriser l'ECG afin de détecter une augmentation de la fréquence cardiaque qui surviendrait si l'épinéphrine était injectée dans une veine épidurale. Ils ont recommandé l'utilisation de $15 \mu \mathrm{g}$ d'épinéphrine. Des publications subséquentes n'ont pas approuvé cette approche à cause 1) des effets néfastes possibles de l'épinéphrine sur le flot sanguin utérin ${ }^{14}$ et 2) de la difficulté d'interpréter une augmentation de la fréquence cardiaque chez les parturientes pouvant manifester 20-30 pour cent de changement de fréquence cardiaque lors des contractions. ${ }^{15}$

Dans ce numéro l'article de revision publié par Dain, Rolbin et Hew discute en détails des problèmes de sélection et d'interprétation de la dose épidurale d'essaj. Je suis d'accord avec la conclusion des auteurs que ba lidocaïne est le meilleur choix parmi les anesthésiques locaux actuellement disponibles. La lidocaïne provoquera un bloc sensoriel et moteur dans un lapse de temps plus court que la bupivacaïne si elle est injectée dans l'espace sous-arachnoidient. Je pense aussi que la solution hyperbare de lidocaine poumrait, si disponible, être plus sécuritaire car elle limiterait l'extension dans l'espace sous-arachnoidient. L'utilisation de la dose d'essai afin de démontrer une injection intravasculaire est beaucoup moins fiable et l'utilisation de l'épinéphrine comme marqueur peut nous confondre chez une patiente en travail.

Comme Dain et al. j'utilise les doses d'essai. Je considère que la dose d'essai est principalement un indicateur d'une injection sous-arachnoïdienne. Actuellement après un test d'aspiration négatif j'injecte $2.0 \mathrm{ml}$ de lidocaïne deux pour cent à travers le cathéter, j"attends cinq minutes et je vérifie le bloc moteur et sensoriel. La pression artérielle ainsi que la fréquence cardiaque sont surveillées aux deux minutes. J'interroge aussì la patiente sur l'engourdissement autour des lèvres et le bourdonnement des oreilles comme des indices d'une injection intravasculaire. Ceci, tout en sachant que l'absence de ces symptômes n'élimine pas une injection intravasculaire.

$\mathrm{Si}$ aucun des signes ou symptòmes néfastes n'est survenu j'injecte soit de la lidocainne carbonatée pour une césarienne ou de la bupivacaïnc 0.25 pour cent pour les parturientes dans des volumes de 5-6 $\mathrm{ml}$ répétés aux cing minutes. Cette subdivision de la dose représente une autre précaution contre l'injection intravasculaire ou sousarachnoidienne de grande quantité d'anesthésique local. Un bloc satisfaisant doit survenir à l'intérieur de vingt minutes sinon une mauvaise position du catheter est hautement suspecte.

Afin de prévenir particulièrement les injections intravasculaires je n'injecte jamais dans des cathéters contenant du sang apparaissant spontanément ou après aspiration. Si du sang a été obtenu je retire le cathéter et je répète la technique à un autre niveau. Si aucune quantité de sang $n$ 'a été obtenue lors de la seconde insertion j'ajoute alors $15 \mu \mathrm{g}$ d'épinéphrine à la dose d'essai et je surveille la 
fréquence cardiaque soit par l'E.C.G. ou le "pulse oximeter". Si aucune augmentation de la fréquence cardiaque au-delà de 20 batt $\cdot$ min $^{-1}$ ne survient en cinq minutes je continue la procédure comme ci-haut mentionné.

Pour des parturientes devant avoir des "doses de rajout", les doses d'essai sont moins aptes à détecter un problème. Afin d'eviter une injection intravasculaire ou sous-arachnoïdienne je divise les doses de façon à ce que le volume injecté ne soit jamais supérieur à $5 \mathrm{ml}$ avec une attente de trois à cinq minutes entre chaque injection. A chaque injection épidurale on doit avoir une observation constante de la patiente. Les signes vitaux doivent être surveillés chaque une à deux minutes pour au moins dix minutes après chaque injection et les signes d'un bloc moteur et sensitif ne doivent être activement recherchés.

Avec une anesthésie épidurale ou une analgésie obstétricale il n'y a aucun substitut à une observation étroite de la patiente par quelqu'un entraîné à détecter les effets secondaires. La dose d'essai peut aider l'observateur entrainé à détecter les injections intravasculaires ou sous-arachnoïdiennes mais ne doit jamais être substituée à une observation vigilante. Parce que les injections intravasculaires ou sous-arachnoïdiennes de l'anesthésique local sont rares lors de l'anesthésie épidurale, plusieurs anesthésistes peuvent être bercés dans une fausse sécurité à l'idée qu'elles ne surviennent jamais et que des précautions élaborées ne sont pas nécessaires. Ceci peut occasionner une morbidité sévère ou une mortalité pour le cas où une complication survienne. L'anesthésie épidurale peut uniquernent être sécuritaire pour toutes les patientes si chacun d'entre nous assume une attitude respectueuse pour ces complications rares mais sévères.

\section{References}

1 Doglioti $A M$. Segmental peridural anesthesia. Am J Surg 1933; 20; 107-18.

2 Madden FC. Jonnesco's method of spinal anesthesia. Br Med J 1910: 2: 914.

3 Eisen SM, Rosen N, Winesanker $H$ er al. Routine use of lumbar epidural anesthesia in obstetrics: A clinical review of 9,532 cases. Can Anaesth Soc J 1960 7: 280-9.

4 Bonica $J J$. Continuous peridural block. Anesthesiology 1956; 17: 626-32.

5 Bromage $P R$. Continuous lumbar epidural analgesia for obstetrics. Can Med Assoc J 1961: 85: 1136-9.

6 Hellman $K$. Epidural anaesthesia in obstetrics: a second look at 26,127 cases. Can Anaesth Soc J 1965; 12: 398-404.

7 Crawford $\cdot J S$. Some matemal complications of epidural anaesthesia for labour. Anaesthesia 1985; 40: 1219-25.

8 Scott DB. Analgesia in labour. Br J Anaesth 1977; 49: 11-7.

9 Prince $G, M c G r e g o r D$. Obstetric epidural test doses Anaesthesia 1986; 41: 1240-50.

10 Albright GA. Cardiac arrest following regional anesthesia with etidocaine or bupivacaine. Anesthesiology 1979; 51: 285-7.

11 DeJong RH, Ronfeld RA, DeRosa RA. Cardiovascular effects of convulsant and supraconvulsant doses of amide local anesthetics. Anesth Analg 1982; 61: 3-9.

12 Kotelcko DM. Shnider SM, Dailey PA et al. Bupivacaine induced cardiac arthythmias in sheep. Anesthesiology 1984; 60: $10-8$.

13 Moore DC, Batra MS. The component of an effective test dose prior to epidural block. Anesthesiology 1981; 55: 693-9.

14 Hood DD, Dewan DM, Rose JC, James III FM. Matemal and fetal effects of intravenous epinephrine containing solutions in gravid ewes. Anesthesiology 1983; 59: A393.

15 Cartwright PP, McCarroll SM, Antzaka C. Maternal heart rate changes with a plain epidural test dose. Anesthesiology 1986; 65: 226-8. 